\title{
Quantum Pair Creation of Soliton Domain Walls
}

\author{
J. H. Miller, Jr. 固 G. Cárdenas, A. García Pérez, W. More, and A. W. Beckwith \\ Department of Physics and Texas Center for Superconductivity and Advanced Materials \\ University of Houston \\ Houston, Texas 77204-5005, USA
}

(Dated: March 13, 2018)

\begin{abstract}
A large body of experimental evidence suggests that the decay of the false vacuum, accompanied by quantum pair creation of soliton domain walls, can occur in a variety of condensed matter systems. Examples include nucleation of charge soliton pairs in density waves [eg. J. H. Miller, Jr. et al., Phys. Rev. Lett. 84, 1555 (2000)] and flux soliton pairs in long Josephon junctions. Recently, Dias and Lemos [J. Math. Phys. 42, 3292 (2001)] have argued that the mass $m$ of the soliton should be interpreted as a line density and a surface density, respectively, for $(2+1)-\mathrm{D}$ and $(3+1)-\mathrm{D}$ systems in the expression for the pair production rate. As the transverse dimensions are increased and the total mass (energy) becomes large, thermal activation becomes suppressed, so quantum processes can dominate even at relatively high temperatures. This paper will discuss both experimental evidence and theoretical arguments for the existence of high-temperature collective quantum phenomena.
\end{abstract}

PACS numbers: 03.75.Lm, 71.45.Lr, 75.30.Fv , 85.25.Cp, 11.27.+d

\section{BACKGROUND AND MOTIVATION}

A wide class of nonperturbative phenomena in field theory can be understood in terms of quantum tunneling. A well-known example is the quantum decay of the false vacuum 1], which has been of broad scientific interest in cosmology 2, 3] and other fields [4] for over two decades. In three dimensions, the boundary between the bubble of true vacuum and the surrounding false vacuum is a type of topological defect known as a domain wall. A variety of topological defects in condensed matter systems have been proposed to nucleate via quantum or thermal fluctuations. These include vortex-antivortex pairs and vortex rings in superconductors 5, 6], superfluids [7, 8], and Bose-Einstein condensates [9]; dislocation pairs in Wigner crystals [10] and vortex lattices 11]; phase-slip vortex rings in charge density waves (CDWs) [12, 13]; charge (or flux) soliton-antisoliton pairs in density waves 14, 15] (or Josephson junctions 16]); and soliton-like domain walls surrounding cigar-shaped bubbles of true vacuum in three-dimensional CDWs [17].

Topological defects, such as flux vortices, play an especially important role in the cuprates and other type-II superconductors. Magnetic relaxation rates that depend weakly on temperature up to $20 \mathrm{~K}$ [18], or even decrease with temperature 19], suggest that vortices may tunnel over a wide temperature range. Moreover, the consistently low $I_{c} R_{n}$ products of cuprate Josephson devices suggest that Josephson vortex-antivortex pair creation [16] may occur when the current is much smaller than the "classical" critical current $I_{0} \sim \Delta / R_{n} e$.

A charge density wave $(\mathrm{CDW})$ is a condensate [20]

*Electronic address: jhmiller@uh.edu in which the electronic charge density in a quasi-onedimensional metal is modulated, $\rho(x, t)=\rho_{0}(x, t)+$ $\rho_{1} \cos \left[2 k_{F} x-\phi(x, t)\right]$. Here $\rho_{0}(x, t)$ contains the background charge of the condensed electrons, and an excess or deficiency of charge proportional to $\pm \partial \phi / \partial x$. A spin density wave (SDW) has a modulated spin density, and is equivalent to two out-of-phase CDWs for the spin-up and spin-down subbands. Although pinned by impurities, a density wave (DW) can transport a current when an applied field exceeds a threshold value $E_{T}$. The most widely studied models of DW depinning are variations of that proposed by Fukuyama, Lee, and Rice 21].

A long-standing debate concerns whether a classical description is sufficient to describe density wave depinning, or a quantum treatment is required. John Bardeen [22, 23] proposed a model in which condensed, dressed electrons Zener tunnel through a "pinning gap," whose energy per electron is small compared to the Peierls gap. This model was motivated by the Zener-like behavior, $J \sim\left[E-E_{T}\right] \exp \left(-E_{0} / E\right)[24,25]$ observed in the current-field characteristics of $\mathrm{NbSe}_{3}$ and $\mathrm{TaS}_{3}$. Bardeen pointed out that the $3-\mathrm{D}$ coherence of the DW condensate would suppress thermal excitations without necessarily suppressing the amplitude for coherent tunneling of the internal microscopic degrees of freedom. The observed Zener field $E_{0}$ does not increase with the transverse dimensions, which has important implications for any theory based on tunneling probabilities.

Maki [14] considered a real-space picture, in which $2 \pi$-soliton-antisoliton $\left(S-S^{\prime}\right)$ pairs nucleate via quantum tunneling. Krive and Rozhavsky [26] pointed out the existence of a Coulomb blockade threshold field for the creation of charged $S-S^{\prime}$ pairs, and later extended this picture to nucleation of soliton-like domain walls [17. A more recent paper [27] proposed an analogy to time-correlated single electron tunneling to explain 
the observed lack of DW polarization below threshold, and to interpret key features of DW dynamics, such as coherent oscillations and narrow-band noise. Observations of Aharonov-Bohm oscillations [28] in the magnetoconductance of CD Ws in $\mathrm{NbSe}_{3}$ crystals with columnar defects strongly support the idea that quantum transport plays a fundamental role. Additional evidence for quantum behavior in density waves is found in $r f$ experiments 29, 30] that show very good agreement with photon-assisted tunneling theory at temperatures up to $215 \mathrm{~K}$.

It is important to also consider whether or not density wave dynamics can be interpreted classically. Perhaps the most obvious requirement for classical sliding is that the washboard potential should be tilted enough for the deformable object to slide over the pinning barrier. This tilting of the washboard potential leads to a predicted phase displacement of $\pi / 2$ in the s- $G$ model at the classical threshold, and to even greater predicted phase displacements when disorder is included [31]. However, NMR and high-resolution x-ray scattering experiments show that the phase displacements of both charge 32, 33, 34 and spin [35] density waves are more than an order of magnitude smaller than classically predicted values near threshold.

Classical models also predict [31, 36] that the lowfrequency dielectric response should increase, or even diverge, as the classical threshold is approached from below. Such predictions are refuted by the observed biasindependent $r f$ and microwave responses below threshold in both CDWs 30, 37] and Wigner crystals 38]. These experiments strongly suggest that the washboard pinning potential is barely tilted as the measured threshold field is attained. Thus, the observed threshold appears to be much smaller than the classical depinning field, and may represent a Coulomb blockade threshold for the creation of charged topological defects. Moreover, attempts 39. using a scanning tunneling microscope (STM) to directly observe either displacement of the CDW below threshold or sliding above threshold have been unsuccessful. The apparent lack of sliding seen in STM experiments and the jerky dynamics revealed by NMR experiments [32, 33. suggest that the DW spends most of its time in the pinned state even above threshold.

The classical deformable DW model also predicts that the high-field $d c$ conductivity should have the form $\sigma_{c d w} \sim a-b E^{-1 / 2}$, where $a$ and $b$ are constants [40]. This prediction is contradicted by experiments [41] that show substantial departure from the classically predicted behavior, but essentially perfect agreement with the Zener form $\sim \exp \left(-E_{0} / E\right)$, in the high-field limit. Coppersmith and Littlewood [42] (CL) make the following predictions for mode locking of a moving DW to an $a c$ source within the classical model. (1) Damped relaxation is crucial in that complete mode locking can occur only when the $a c$ frequency and amplitude are small enough for significant relaxation to take place when the total field is less than $E_{T}$. (2) For large $a c$ amplitudes and frequen- cies such that the time interval spent below threshold is small, the differential resistance $d V / d I$ should exhibit peaks but not complete mode locking. In addition, no "wings" (sharp negative dips in $d V / d I$ adjacent to the interference peak) should be observed. These predictions are refuted incontrovertibly by $a c-d c$ interference and mode locking experiments on high quality $\mathrm{NbSe}_{3}$ crystals 41] that demonstrate both negative wings and complete mode locking at high $d c$ bias fields and high frequencies.

The failure of CLs calculations to account for the mode-locking experiments reflects a fundamental deficiency of the classical deformable model. When the DW slides rapidly, relaxation into the pinning potential wells cannot occur quickly enough to prevent the deformations and net pinning energy from vanishing. The characteristic frequency for this relaxation, the DW dielectric relaxation frequency, is measured to be about $5 \mathrm{MHz}$ in $\mathrm{NbSe}_{3}$ [43]. The observations of complete mode locking at $a c$ and drift frequencies of up to $1 \mathrm{GHz}$ indicate that the strength of DW pinning is undiminished in a regime where significant relaxation cannot occur within the conventional classical model. Thus, quantum mechanisms must be explored to understand, fundamentally, DW depinning and dynamics.

\section{SOLITON TUNNELING MODEL}

A realistic density wave pinning potential would have the form $U_{0}(\mathbf{r})\left\{1-\cos \left[\phi-\phi_{0}(\mathbf{r})\right]\right\}$, which includes spatial variations of both the pinning energy $U_{0}$, and the optimum phase $\phi_{0}$. However, observations of narrow band noise, coherent oscillations, and complete mode-locking with an ac source [1] in high quality $\mathrm{NbSe}_{3}$ crystals suggest that both $U_{0}$ and $\phi_{0}$ are slowly varying in weakly pinned DWs. The sine-Gordon (s-G) model thus provides an idealized conceptual framework for interpreting many aspects of density wave transport 44], from both classical and quantum points of view. Similarly, a weakly-coupled Josephson junction (JJ) can be described by a phase, $\phi(x, t)$, representing the phase difference across the junction, in a sine-Gordon potential.

Density waves and JJs are dual in that the roles of charge and flux are interchanged, as well as those of current and voltage. The current in a density wave is $I=\left(Q_{0} / 2 \pi\right) \partial \phi / \partial t$, where $Q_{0} \sim 2 e N_{c h}$ and $N_{c h}$ is the number of parallel chains, whereas the voltage across a JJ is $V=\left(\Phi_{0} / 2 \pi\right) \partial \phi / \partial t$, where $\Phi_{0}=h / 2 e$. Charge (flux) solitons in a density wave (JJ) carry a charge (flux) of $\pm Q_{0}\left( \pm \Phi_{0}\right)$ (see Table 【). The width of a Josephson vortex is roughly the Josephson penetration length, $\lambda_{J} \propto J_{c}^{-1 / 2}$ whereas, in a DW, the soliton width is $\lambda_{0}=c_{0} / \omega_{0}$, where $c_{0}$ is the phason velocity and $\omega_{0}$ is the pinning frequency. Thus, $\lambda_{0}$ will increase with decreasing impurity concentration (as $\omega_{0}$ decreases), and may approach the distance between contacts in extremely pure samples. This is equivalent to approaching the short junction limit, $L<\lambda_{J}$, in a JJ, where the $V-I$ curves 
TABLE I: Charge-flux duality between density waves and Josephson junctions.

\begin{tabular}{lcc}
\hline \hline & Density Wave & Josephson junction \\
Soliton or antisoliton & Kink w/ charge $\pm Q_{0}$ & Josephson vortex w/ flux $\pm \phi_{0}$ \\
Type of threshold & Threshold field $E_{T}$ & Threshold current $I_{T}$ \\
Transport characteristic & $I$ vs. $V, I=2 \pi Q_{0} \partial \phi / \partial t$ & $V v s . I, V=2 \pi \phi_{0} \partial \phi / \partial t$ \\
\hline \hline
\end{tabular}

become significantly less rounded.

The quantum decay of the metastable false vacuum of a scalar field $\phi$, accompanied by the creation of solitons and antisolitons in $(1+1)$ dimensions [45, 46, 47] has been studied extensively in quantum field theory. Dias and Lemos 48] (DL) have calculated the effective one loop action for pair creation of solitons in the $(1+1)-\mathrm{D}$ s-G model. In addition, they have extended their arguments to pair creation of soliton domain walls in higher dimensions. In the $(1+1)-\mathrm{D}$ case, the soliton-antisoliton pair production rate per unit time and length, $\Gamma / L$, is identified with the decay rate of the false vacuum. This is calculated to be, in the absence of Coulomb interactions between the solitons (taking $\hbar=c=1$ ) [48]:

$$
\frac{\Gamma}{L}=\frac{\varepsilon}{2 \pi} \exp \left[\frac{-\pi m^{2}}{\varepsilon}\right]
$$

where $m$ is the soliton mass (energy). Here $\varepsilon$ represents the gain in energy per unit length between the two created solitons, which is proportional to the applied electric field $E$ for a density wave (and to the applied current for a JJ). The energy for pair creation is contained in the line between the particles and is given by $2 x \varepsilon=2 m$, where $2 x$ is the distance between the solitons.

DL point out [4] that a one-particle system in $(1+1)-\mathrm{D}$ can be transformed into a line in $(2+1)-\mathrm{D}$ and a thin wall in $(3+1)-\mathrm{D}$, except that the mass $m$ should be interpreted as a line density and a surface density, respectively. Their calculations thus apply directly to the domain wall pair creation. As the transverse dimensions are increased and the total mass (energy) becomes large, thermal activation becomes suppressed, so quantum processes can dominate even at relatively high temperatures (up to $400 \mathrm{~K}$ for the CDW in $\mathrm{NbSe}_{3}$ ).

As an example, consider the $(2+1)$-D case, where one can make a cut normal to the infinite strings so that " $\varepsilon$ " will still be the energy density of the line between the two infinitesimal pieces of the strings. Each one of these infinitesimal pieces has a mass $m$, so $m$ represents the line density of the string. The energy for pair creation of strings comes from the infinite plane between the two strings, and is given by $2 x d \varepsilon=d 2 \mathrm{~m}$, where $d$ is the length of the string, which goes to infinity [49]. Therefore both the mass of the string and the energy to create the pair of strings become infinite.

Importantly, " $m$ " can no longer be interpreted as the total mass of the string or of the domain wall. Indeed such an interpretation would correspond to creation of point particles in $(2+1)$-D or in $(3+1)-\mathrm{D}$, where the total mass $m$ would be infinite. The infinite energy for pair creation would then have to come from the line between the two particles rather than from the infinite plane between the two strings. DLs calculations do not apply to this process. In the generalization to higher dimensions, one is using the symmetry of the system along the extra direction. In Minkowski space-time, the procedure is direct [49]. However, in systems that include gravitation, the procedure is usually not so straightforward, and one must employ dimensional reduction or dimensional expansion [50].

Suppose we now put the constants $\hbar$ and $c$ back into Eq. 1 and take $\lambda$ to represent the Compton wavelength, $\lambda=\hbar / m c$. (In a density wave, the effective "speed of light" is the phason velocity $c_{0}$, which is about $3 \times 10^{5}$ $\mathrm{cm} / \mathrm{s}$.) Explicitly putting the physical constants into the exponent, we then have: $m \pi^{2} / \varepsilon \rightarrow m \pi c^{2} /[q E \lambda]$, where $q$ is the soliton charge for a single chain (i.e. for the $(1+1)-\mathrm{D}$ system) and $E$ is the electric field. (In a JJ, the charge and electric field would be replaced by the flux $\Phi_{0}$ and the current density.) For the $(3+1)-\mathrm{D}$ system, we can now define $m^{\prime}=m / a^{2}$ and $q^{\prime}=q / a^{2}$ to be the mass and charge per unit area of the soliton domain wall, respectively. In a density wave, the length represents the transverse distance between DW chains. Note that, for the exponent $\pi m^{\prime} c^{2} /\left[q^{\prime} E \lambda\right]$ to remain dimensionless, $\lambda$ needs to retain its original units of length. One way of doing this is to define a new "quantum of action per unit area" in terms of the characteristic length scale $a$, i.e. $\hbar^{\prime}=\hbar / a^{2}$, leading to a Compton wavelength, $\lambda=\hbar^{\prime} / m^{\prime} c$, which has the same value as before. Lattice gauge theorists have recently examined models of domain wall fermions (bosons), whose anticommutators (commutators) differ from those of point particles, thus lending credence to the arguments presented here.

Another way of looking at the problem is to recall that $\hbar$ has units of angular momentum: $L=r m v$, where $r$ = distance, $m=$ mass, and $v=$ velocity. Now, since the mass transforms as $m^{\prime}=m / A$, where $A$ is an area, from $L=r m v$ we must have $L^{\prime}=L / A$, so that $\hbar^{\prime}=$ $\hbar / A$ 49]. The commutator between bosonic domain wall creation and annihilation operators will then be scaled accordingly. The Compton wavelength of the $(3+1)-\mathrm{D}$ problem refers to the direction normal to the domain 
wall, and is thus $\lambda=\hbar^{\prime} / m^{\prime} c=\hbar / m c$.

It should be noted that $r f$ experiments [25, 29, 30] are consistent with a ratio $\hbar / q=\hbar^{\prime} / q^{\prime}$ that is scale invariant, where $q=2 e$ for a fully condensed CDW. This is further supported by magneto-transport experiments 28], which yield Aharonov-Bohm oscillations with a periodicity of $h / 2 e$, and not $h / 2 N e$ as predicted theoretically [51], where $N$ is the number of coupled chains. However, the observed mode locking with an $a c$ source 41] shows that the phase is coherent throughout macroscopic regions within the crystal, suggesting quantum nucleation of entire domain walls rather than just single dislocations.

The above arguments lead, for a CDW, to a Zener-like current-field characteristic given by:

$$
J \sim E \exp \left[-E_{0} / E\right]
$$

where the characteristic Zener field $E_{0}=\left[\pi m^{\prime 2} c_{0}^{3}\right] /\left[\hbar^{\prime} q^{\prime}\right]$ is independent of the traverse dimensions. (For a JJ, the $V$ vs. $I$ curve would be given by $V \sim I \exp \left[-I_{0} / I\right]$.) DLs calculations neglect Coulomb interactions between charge solitons (or magnetic interactions between flux solitons in a JJ), and thus fail to predict a sharp threshold field (threshold current for a JJ) for pair creation. The following section examines the origin of the threshold field as a macroscopic Coulomb blockade effect (or its dual for the case of a JJ).

\section{MACROSCOPIC COULOMB BLOCKADE AND TIME-CORRELATED SOLITON TUNNELING}

The quantum interpretation of the threshold field, as a pair-creation threshold due to Coulomb blockade, is motivated by Colemans paper [52] on soliton pair-creation in the massive Schwinger model. A pair of $S$ and $S^{\prime}$ domain walls with charges $\pm Q_{0}$ produce an internal field of magnitude $E^{*}=Q_{0} / \varepsilon A$, as shown in Fig. 1] where $A$ is the cross-sectional area and $\varepsilon$ is the dielectric constant. When a field $E$ is applied, the difference in electrostatic energies of a state with a pair of separation $l$ and of the "vacuum" is $\Delta U=(1 / 2) \varepsilon A l\left[\left(E+E^{*}\right)^{2}-E^{2}\right]=$ $Q_{0} l\left[(1 / 2) E^{*} \pm E\right]$, which is positive when $|E|<(1 / 2) E^{*}$. Conservation of energy thus forbids pair production for fields less than a quantum threshold, $E_{T} \equiv(1 / 2) E^{*}=$ $Q_{0} / 2 \varepsilon A$, which appears to be about two orders of magnitude smaller than the classical depinning field in $\mathrm{NbSe}_{3}$ 27. The observed universality relation, $\varepsilon E_{T} \sim e N_{c h} / A$ [20], thus arises quite elegantly in this model. Screening by the uncondensed normal carriers greatly enhances the dielectric response of $\mathrm{NbSe}_{3}$, which has an incomplete Peierls gap and also has a much lower threshold field than most other CDW materials. (In a JJ, charge-flux duality leads to a quantum threshold current proportional to $\Phi_{0} / 2 L_{M}$, where $L_{M}$ is the mutual inductive coupling between the Josephson vortex and the antivortex.)

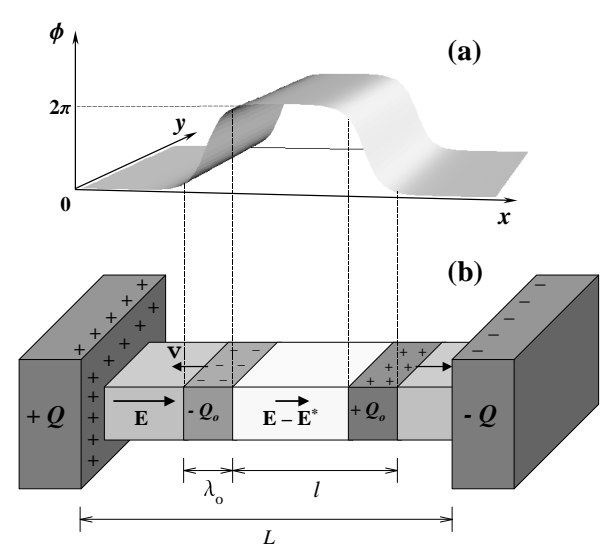

FIG. 1: (a) Density wave phase vs. position, illustrating the production of a soliton-antisoliton domain wall pair. (b) Model of a density wave capacitor, showing the nucleated domain walls moving towards the contacts. The electric field between the domain walls is reduced by the internal field $E^{*}$. The distances $l, \lambda_{0}$, and the crystal thickness are greatly exaggerated for clarity.

A density wave between two contacts behaves as a capacitor with an enormous dielectric constant, as shown in Fig. 1] The initial charging energy is $Q^{2} / 2 C$, where $Q$ is the displacement charge and $C=\varepsilon A / L$. We define $\theta \equiv 2 \pi Q / Q_{0}=2 \pi E / E^{*}=\pi E / E_{T}$ and note that a displacement $\phi$ near the middle creates a non-topological kink-antikink pair, with charges $\pm(\phi / 2 \pi) Q_{0}$, if $\phi=0$ at the contacts. The washboard pinning and quadratic charging energies can then be written as [27]:

$$
U[\phi]=\int_{0}^{L} d x\left\{u_{p}[1-\cos \phi(x)]+u_{c}[\theta-\phi(x)]^{2}\right\}
$$

where the first and last terms represent the pinning and electrostatic charging energies, respectively, and where $u_{p} \gg u_{c}$ for $\mathrm{NbSe}_{3}$. If the system starts out in its ground state, conservation of energy will prevent tunneling when the applied field is below threshold, $\theta<\pi\left(E<E_{T}\right)$, as illustrated in Fig. 2. However, when $\theta$ exceeds $\pi$, what was formerly the true vacuum becomes the unstable false vacuum. One or more bubbles of true vacuum, with soliton domain walls at their surfaces, then nucleate and expand rapidly (Fig. I). After $n$ solitons of charge $Q_{0}$ (and antisolitons of charge $-Q_{0}$ ) have reached the contacts, the charging energy becomes:

$$
\frac{\left(Q-n Q_{0}\right)^{2}}{2 C}=\frac{Q_{0}^{2}}{8 \pi^{2} C}(\theta-2 \pi n)^{2}
$$

This series of piecewise parabolas is similar to the charging energy of a single-electron tunnel junction, except that $Q_{0}$ now represents a macroscopic charge. 

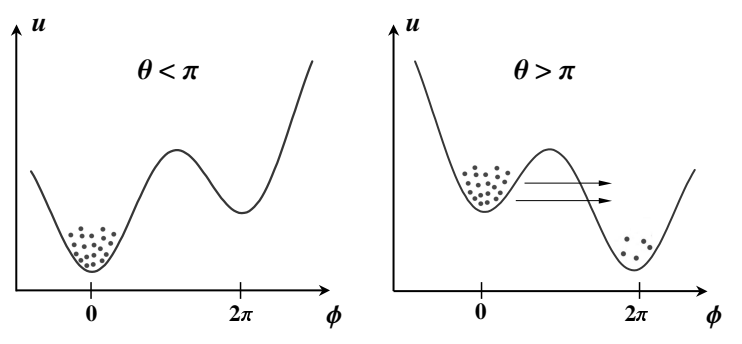

FIG. 2: Plot of potential energy vs. $\phi$ for two different values of $\theta$, in which the many spatial degrees of freedom are illustrated schematically. Tunneling is prevented by conservation of energy when $\theta<\pi$. When $\theta>\pi$, parts of the system tunnel into the adjacent well via decay of the false vacuum.

The single-electron transistor (SET) 53 consists of a gate capacitor $C_{g}$ coupled to an island electrode between two small capacitance tunnel junctions in series. The gate voltage modulates the $I-V$ curves between the source and drain electrodes, with a period $e$ in displacement charge, $Q_{g}=C_{g} V_{g}$. The displacement charges $Q_{1,2}$ across the two tunnel junctions are related as $Q_{2}=Q_{1}+Q_{g}+q_{0}$, where $q_{0}$ is a phenomenological offset charge induced during cooling 53. The SET is related by charge-flux duality to the dc SQUID. The critical voltage across an SET is a periodic function of $Q_{g}$, whereas the critical current across a SQUID is periodically modulated (with period $\Phi_{0}$ ) by the flux $\Phi$.

The model discussed above suggests that it may be possible to demonstrate a macroscopic version of the SET, by attaching a gate capacitor to an island electrode near the center of a quasi-1-D crystal with a density wave. The displacement charge induced by the gate electrode would then periodically modulate the total critical voltage between the source and drain electrodes. Ideally, in the absence of any shunt conductance, the periodicity of the gate displacement charge might be expected to be $\sim Q_{0}$. However, screening by the normal, uncondensed electrons will tend to reduce the effectiveness of the gate which, unlike the source and drain contacts, cannot be driven by a current source. The displacement charges across the two segments of the crystal will be related as $Q_{2}=Q_{1}+\beta\left(Q_{g}+q_{0}\right)$, where $\beta \ll 1$ reflects screening by the normal carriers. The total charging energy of the two segments, in this idealized model, will then be:

$$
\frac{\left(Q_{1}-n_{1} Q_{0}\right)^{2}}{2 C_{1}}+\frac{\left(Q_{2}-n_{2} Q_{0}\right)^{2}}{2 C_{2}}=\frac{Q_{0}^{2}}{8 \pi^{2} C}\left\{\frac{\left(\theta_{1}-2 \pi n_{1}\right)^{2}}{2 C_{1}}+\frac{\left(\theta_{2}-2 \pi n_{2}\right)^{2}}{2 C_{2}}\right\}
$$

where $C_{1}$ and $C_{2}$ are the capacitances of the two segments separated by the island electrode. The analogy to the SET suggests that a gate voltage might modulate the $I-V$ curves between source and drain contacts, with a periodicity $\Delta V_{g} \sim Q_{0} / \beta C_{g}$. The gate capacitance $C_{g}$, and hence the attainable displacement charge $Q_{g}$, may have been too small to observe non-monotonic behavior in previous experiments [54], in which a gate electrode was fabricated directly on the crystal to form a MOSFET-like structure. The soliton tunneling transistor (STT), discussed in the next section, employs a much larger, $1 \mu \mathrm{F}$, gate capacitor coupled to an $\mathrm{NbSe}_{3}$ crystal, and exhibits non-monotonic behavior.

An exact calculation of the charging energy would yield a plot in which the energy is reduced slightly at the crossing points, $\theta=n \pi$, as compared to piecewise parabolas. However, Eq. 4 provides a reasonable approximation to $E(\theta)$ :

$$
E(\theta) \sim(\theta-2 \pi n)^{2}
$$

As in the SET, the voltage across each segment of the STT is related to the charging energy as $V_{1,2}=$ $d E / d Q_{1,2} \propto d E / d \theta_{1,2}$. If we use the approximation given by Eq. [6] this yields a sawtooth function, which can be expanded as a Fourier series:

$$
V_{1,2}=V_{0} \operatorname{saw}\left(\theta_{1,2}\right)=-\frac{V_{0}}{\pi} \sum_{n=1}^{\infty} \frac{(-1)^{n}}{n} \sin \left(n \theta_{1,2}\right)
$$

This model can also been used to model the dynamics of density waves and to interpret the narrow-band noise spectra, the many harmonics of which are consistent with a sawtooth function.

Equation 7 is related by charge-flux duality to the current-phase relation of a Josephson junction. The periodic behavior of the source-to-drain voltage vs. gate voltage of an STT can readily be understood by exploiting the duality with a dc SQUID. When each JJ in a dc SQUID has an ideal, sinusoidal current-phase relation and the critical currents $I_{0}$ are identical, the total current is $I=I_{0}\left[\sin \varphi_{1}+\sin \varphi_{2}\right]$, where $\varphi_{2}=\varphi_{1}+2 \Phi / \Phi_{0}$. This yields a total critical current, $I_{c}=2 I_{0}\left|\cos \left(2 \pi \Phi / \Phi_{0}\right)\right|$, which is a periodic function of the flux $\Phi$. Similarly, the total source-to-drain voltage of an ideal STT, $V=$ $V_{1}+V_{2}$, where $V_{1,2}$ are given by Eq. 7 yields a critical voltage that is a periodic function of gate voltage $V_{g}=Q_{g} / C_{g}$, as shown in Fig. B] Here we note that $\theta_{2}=\theta_{1}+\theta_{g}+\theta_{0}$, where $\theta_{g}=2 \pi \beta Q_{g} / Q_{0}=2 \pi \beta q_{0} / Q_{0}$, $Q_{g}$ is the displacement charge across the gate capacitor, 


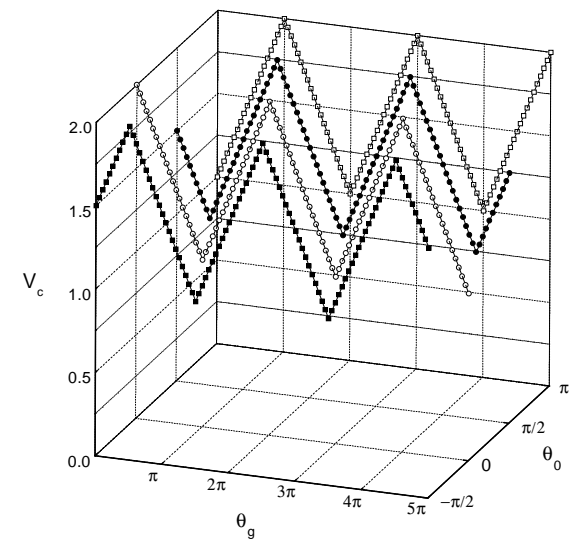

FIG. 3: Predicted critical voltage vs. normalized gate voltage $\theta_{g}$ for several values of $\theta_{0}$ using the idealized model discussed in Section III, showing the periodic behavior.

$q_{0}$ is the offset charge, and $\beta$ is the screening parameter due to the normal electrons.

\section{SOLITON TUNNELING TRANSISTOR EXPERIMENT}

The experiment reported here employs a geometry analogous to that of the SET, in which the "source-todrain" $I-V$ characteristic is modulated by a voltage applied to a gate capacitor. Single crystals of $\mathrm{NbSe}_{3}$ were employed in the experiment. This material forms two independent CDWs, at Peierls transition temperatures of $145 \mathrm{~K}$ and $59 \mathrm{~K}$ 24], respectively. The Peierls gap opens up over most of the Fermi surface (FS) below the lower transition, but leaves a small portion of the FS intact, so that a significant concentration $\left(\sim 6 \times 10^{-18} \mathrm{~cm}^{-3}\right)$ of normal, uncondensed carriers remain down to low temperatures.

The geometry used in our experiment is illustrated in the inset to Fig. [4 where the width of the crystal is exaggerated for clarity. The $\mathrm{NbSe}_{3}$ crystal was placed onto an alumina substrate with a series of evaporated, $25 \mu \mathrm{m}$ wide gold contacts. The substrate was thermally anchored to a cold-finger in the vacuum shroud of an open cycle helium flow cryostat and the temperature was controlled using a Lake Shore temperature controller attached to a heater coil wrapped around the cold-finger. A Keithley programmable $d c$ current source injected the current between two contacts, which were bonded to the crystal near the ends using silver paint. The "source-todrain" voltage was measured between two additional gold contacts, as illustrated, and a $1 \mu \mathrm{F}$ gate capacitor was attached to the center gold contact using silver paint. The spacing between contacts along the crystal was $500 \mu \mathrm{m}$ center-to-center, and the gate capacitor was kept inside the cryostat.

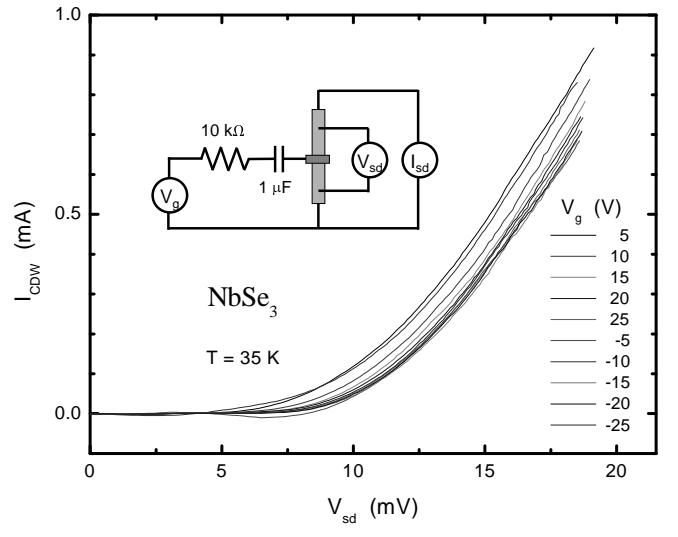

FIG. 4: CDW current vs. source-to-drain voltage in a soliton tunneling transistor $\left(\mathrm{NbSe}_{3}\right)$ for several values of gate voltage at $35 \mathrm{~K}$. (The shunt current of the normal electrons has been subtracted for clarity.)

We found that substantially smaller gate capacitors (as well as gate capacitors with longer leads) were unable to induce a periodic modulation of the $I-V$ characteristic. Moreover, $d c I-V$ (rather than differential $d V / d I)$ measurements were necessary to avoid inducing a displacement current through the gate capacitor. A programmable voltage source was coupled to the gate capacitor via a $10-\mathrm{k} \Omega$ resistor, which limited the current flowing through the crystal during changes in gate voltage when the gate capacitor either partially charged or discharged. The cryostat was kept inside an electromagnetically shielded enclosure, and $V_{s d}$ was measured with a nanovoltmeter.

The measurements were primarily carried out at $35 \mathrm{~K}$. Previous "field effect transistor" experiments 54] showed the greatest modulation at around $30 \mathrm{~K}$, where the threshold field is near its minimum. We also observed the largest modulation, using our geometry, at comparable temperatures. We attained the best temperature stability (better than $\pm 0.01 \mathrm{~K}$ ) when the temperature was set to $35 \mathrm{~K}$, and thus chose this temperature for most of the measurements reported here.

Figure 4 4 shows several plots of CDW current as a function of source-to-drain voltage, $I_{c d w}$ vs. $V_{s d}$, in a $\mathrm{NbSe}_{3}$ crystal at $35 \mathrm{~K}$, for different values of gate voltage $V_{g}$. The gate voltage is seen to modulate the threshold voltage in the $I-V$ curves of Fig. 4. Figure 15 displays plots of source-to-drain voltage $V_{s d} v s$. $V_{g}$ for three values of total bias current above threshold. The plots exhibit roughly periodic behavior, similar to that observed in SETs. However, in our system, the measured periodicity $\Delta V_{g} \sim 10 \mathrm{~V}$ is consistent with a macroscopic displacement charge $\Delta Q=C_{g} \Delta V_{g} \sim 6 \times 10^{13} e$, comparable to the charge of the conducting electrons between the contacts.

The behavior shown in Fig. 5 is quite extraordinary, 


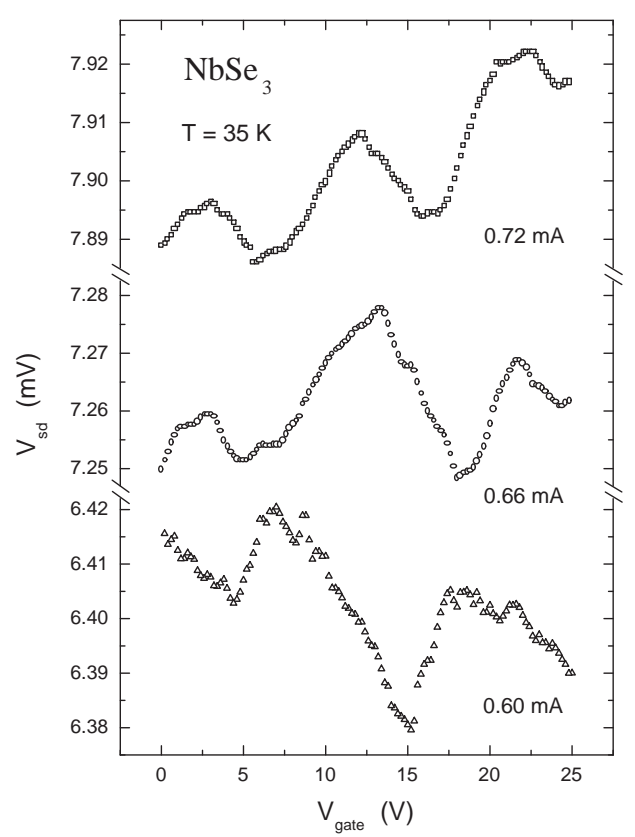

FIG. 5: Source-to-drain voltage $V_{s d}$ vs. $V_{g}$ for fixed values of total bias current at $35 \mathrm{~K}$.

and appears to be consistent with the soliton domain wall tunneling hypothesis. The number of parallel CDW chains, $N_{c h}$, is about $10^{8}$. Thus, one might estimate the screening parameter as follows: $\beta \sim Q_{0} / \Delta Q \sim$ $2 N_{c h} e / \Delta Q \sim 3 \times 10^{-6}$. However, an alternative interpretation for the observed periodicity might be that all of the normal electrons between the contacts participate in screening out the displacement charge $Q_{0}$ of the CDW. Thus, the observation that $\Delta Q / e \sim 6 \times 10^{13}$ is roughly the number of conducting electrons between contacts may not be a coincidence. Further work is needed to better understand the effects of screening by the normal, uncondensed electrons.

\section{IMPLICATIONS}

The implications of the model and experimental results reported here are potentially far-reaching, and could impact fields as diverse as cosmology, condensed matter physics, quantum computation, and biophysics. In the field of cosmology, quantum nucleation of dilaton black hole pairs [55], cosmic strings [56], and even the entire universe 2, 3] have been proposed. The European Science Foundation has recently funded a program, known as Cosmology in the Laboratory (COSLAB), intended to explore laboratory analogs of cosmological objects. Topics being studied include analogies between vortices in superconductors and cosmic strings, and artificial (acoustic or optical) black holes.

In the area of biophysics, Fröhlich suggested [57, 58] that long-range quantum coherence may play a fundamental role in biological systems. This may provide a mechanism to integrate complex interactions that take place within a live cell. A recent model [59] proposes that microtubules, which exist in the cells of all higher organisms but are especially concentrated in neurons, engage in a form of quantum computation. Roger Penrose [60] has even made compelling arguments that large-scale quantum coherence, perhaps mediated by the microtubules, plays a fundamental role in consciousness. It is clear that, for Penroses arguments to be valid, some mechanism is needed to suppress decoherence. Perhaps soliton domain walls, or related topological defects, may prove to be topologically protected from decoherence at biological temperatures. Further research is clearly warranted to explore this extraordinary possibility.

\section{Acknowledgments}

The authors gratefully acknowledge the assistance of James Claycomb, L.-M.(Patrick) Xie, and John McCarten as well as valuable insights provided by Oscar Dias and Jose Lemos. This work was supported, in part, by the State of Texas through the Texas Center for Superconductivity and Advanced Materials and the Texas Higher Education Coordinating Board Advanced Research Program (ARP), and by the R. A. Welch Foundation (E-1221).
[1] S. Coleman, Phys. Rev. D 15, 2929 (1977).

[2] E. P. Tryon, Nature, 246, 396 (1973).

[3] A. D. Linde, Sov. Phys. JETP 60, 211-213 (1984); Zh. Eksp. Teor. Fiz. 87, 369-374 (1984).

[4] A. O. Caldeira and A. J. Leggett, Phys. Rev. Lett. 46, 211 (1981).

[5] J. M. Kosterlitz and D. J. Thouless, J. Phys. C 6, 1181 (1973).
[6] Y. Duan, H. Zhang, and S. Li, Phys. Rev. B 58, 125 (1998).

[7] G. W. Rayfield, and F. Reif, Phys. Rev. 136, A 1194 (1964).

[8] G. E. Volovik, JETP Lett. 15, 81 (1972).

[9] B. P. Anderson, P. C. Haljan, C.A. Regal, D. L., Feder, C. W., Collins, and E. A. Cornell, Phys. Rev. Lett. 86, 2926 (2001). 
[10] S. T. Chui, Phys. Lett. A 180, 149 (1993).

[11] G. Blatter, M. V. Feigelman, V. B. Geshkenbein, A. I. Larkin, and V. M Vinokur, Rev. Mod. Phys. 66, 1125 (1994).

[12] S. V. Zaitsev-Zotov, Phys. Rev. Lett. 71, 605 (1993); S. V. Zaitsev-Zotov, G. Remenyi, and P. Monceau, Phys. Rev. B 56, 6388 (1997).

[13] H. Matsukawa, H. Miyake, M. Yumoto, and H. Fukuyama, J. Phys. IV France 9, Pr10-161 (1999).

[14] K. Maki, Phys. Rev. Lett. 39, 46 (1977); Phys. Rev. B 18, 1641 (1978).

[15] A. Maiti and J. H. Miller, Jr., Phys. Rev. B 4312205 (1991).

[16] A. Widom and Y. Srivastava, Phys. Lett. A 114, 337 (1986).

[17] I. V. Krive and A. S. Rozhavskii, Soviet Physics JETP 69, 552 (1989).

[18] H. H. Wen, R. T. Lu, and S. L. Yan (unpublished).

[19] Y. Y. Xue, Z. J. Huang, P. H. Hor, and C. W. Chu, Phys. Rev. B 43, 13598 (1991).

[20] G. Grüner, Density Waves in Solids, Addison-Wesley, Reading, Mass. (1994).

[21] H. Fukuyama, J. Phys. Soc. Jpn. 41, 513 (1976); H. Fukuyama and P. A. Lee, Phys. Rev. B 17, 535 (1978); P. A. Lee and T. M. Rice, ibid. B 19, 3970 (1979).

[22] John Bardeen, Phys. Rev. Lett. 42, 1498 (1979); ibid. 45, 1978 (1979).

[23] John Bardeen, Phys. Rev. Lett. 55, 1010 (1985).

[24] P. Monceau, N. P. Ong, A. M. Portis, A. Meerschaut, and J. Rouxel, Phys. Rev. Lett. 37, 602 (1976); N. P. Ong and P. Monceau, Phys. Rev. B 16, 3443 (1977).

[25] R. E. Thorne, J. H. Miller, Jr., W. G. Lyons, J. W. Lyding, and J. R. Tucker, Phys. Rev. Lett. 55, 1006 (1985).

[26] I. V. Krive and A. S. Rozhavsky, Solid State Commun. 55, 691 (1985).

[27] J. H. Miller, Jr., C. Ordóñez, and E. Prodan, Phys. Rev. Lett. 84, 1555 (2000).

[28] Yu I. Latyshev, O. Laborde, P. Monceau, and S. Klaumnzer, Phys. Rev. Lett. 78, 919 (1997).

[29] J. H. Miller, Jr., J. Richard, J. R. Tucker, and John Bardeen, Phys. Rev. Lett. 51, 1592 (1983).

[30] J. H. Miller, Jr., R. E. Thorne, W. G. Lyons, J. R. Tucker, and John Bardeen, Phys. Rev. B 31, 5229 (1985).

[31] P. B. Littlewood, Phys. Rev. B 33, 6694 (1986).

[32] J. H. Ross, Jr., Z. Wang, and C. P. Slichter, Phys. Rev. Lett. 56, 663-666 (1986);

[33] J. H. Ross, Jr., Z. Wang, and C. P. Slichter, Phys. Rev. B 41, 2722 (1990).

[34] H. Requardt, F. Ya. Nad, P. Monceau, R. Currat, J. E.
Lorenzo, S. Brazovskii, N. Kirova, G. Grbel, and Ch. Vettier, Phys. Rev. Lett. 80, 5631 (1998).

[35] W. H. Wong, M. E. Hanson, W. G. Clark, B. Alavi, and G. Grner, Phys. Rev. Lett. 72, 2640 (1994).

[36] O. Narayan and A. A. Middleton, Phys. Rev. B 49, 244 (1994).

[37] A. Zettl, and G. Grüner, Phys. Rev. B 29, 755 (1984).

[38] C.-C. Li, L. W. Engel, J. Yoon, D. C. Tsui, and M. Shayegan, J. Phys. IV France 9, Pr10-215 (1999).

[39] G. Gammie, J. S. Hubacek, S. L. Skala, R. T. Brockenbrough, J. R. Tucker, and J. W. Lyding, Phys. Rev. B 40, 11965 (1989).

[40] L. Sneddon, M. C. Cross, and D. S. Fisher, Phys. Rev. Lett. 49, 292 (1982).

[41] R. E. Thorne, J. R. Tucker, and John Bardeen, Phys. Rev. Lett. 58, 828 (1987).

[42] S. N. Coppersmith and P. B. Littlewood, Phys. Rev. Lett. 57, 1927 (1986).

[43] J. H. Miller, Jr., J. Richard, R. E. Thorne, W. G. Lyons, and J. R. Tucker, Phys. Rev. B 29, 2328 (1984).

[44] M. J. Rice, A. R. Bishop, J. A. Krumhansl, and S. E. Trullinger, Phys. Rev. Lett. 58, 828 (1987).

[45] M. Stone, Phys. Lett. B 67, 186 (1977).

[46] M. B. Voloshin, Sov. J. Nucl. Phys. 42, 644 (1985).

[47] V. G. Kiselev, Phys. Rev. D 45, 2929 (1992).

[48] O. J. C. Dias and J. P. S. Lemos, J. Math. Phys. 42, 3292 (2001).

[49] O. J. C. Dias and J. P. S. Lemos, private communication.

[50] J. P. S. Lemos and V. T. Zanchin, Phys. Rev. D 53, 4684 (1996).

[51] E. N. Bogachek, I. V. Krive, I. O. Kulik, and A. S. Rozhavsky, Phys. Rev. B 42, 7614 (1990).

[52] S. Coleman, Annals of Phys. 101, 239 (1976).

[53] M. H. Devoret, and R. J., Nature 406, 1039 (2000).

[54] T. L. Adelman, S. V. Zaitsev-Zotov, and R. E. Thorne, Phys. Rev. Lett. 74, 5264 (1995).

[55] F. Dowker, J. P. Gauntlett, D. A. Kastor, and J. Traschen, Phys. Rev. D 49, 2909 (1994).

[56] R. Basu, A. H. Guth, and A. Vilenkin, Phys. Rev. D 44, 340 (1991).

[57] H. Fröhlich, Nature 228, 1093 (1970).

[58] H. Fröhlich, Proc. Natl. Acad. Sci. USA 72, 4211 (1975).

[59] N. E. Mavromatos and D. V. Nanopoulos, Int. J. Mod. Phys. B 12, 517 (1998).

[60] Roger Penrose, Shadows of the mind: a search for the missing science of consciousness, (Oxford University Press, Oxford, 1994). 